\title{
Management of posterior reversible encephalopathy syndrome in a pediatric patient with a rare adrenocortical tumor after computed tomography-guided biopsy
}

\author{
Ram Singh $^{1}$, Vishal Bhatnagar ${ }^{2}$ \\ From ' Assistant Professor, Department of Oncoanaesthesia and Palliative Medicine, National Cancer Institute, All India Institute of Medical Sciences, \\ New Delhi, India, ${ }^{2}$ Consultant and Head, Department of Surgical Intensive Care Unit, Rajiv Gandhi Cancer Institute and Research Center, Rohini, \\ New Delhi, India
}

\begin{abstract}
The posterior reversible encephalopathy syndrome (PRES) in pediatric patients is rare but a treatable reversible condition. In the pediatric age group, most cases had been reported among patients with leukemia undergoing chemotherapy and rarely in patients with solid tumors. We report a 2-year-old male child with a large retroperitoneal mass arising from the right adrenal gland. The patient had a significant rise in blood pressure followed by seizures and altered sensorium after a computed tomography-guided biopsy from the tumour mass. The patient was managed meticulously considering it as a case of PRES by a multidisciplinary team of the intensive care unit. Later, the adrenal mass was evaluated and confirmed to be a pediatric adrenocortical tumor on biopsy report which is also a rare occurrence in the pediatrics age group.
\end{abstract}

Key words: Adrenocortical tumor, Complications, Posterior reversible encephalopathy syndrome, Seizure

$\mathrm{T}$ The occurrence of posterior reversible encephalopathy syndrome (PRES) in pediatric patients is relatively a rare clinical phenomenon. The reported incidence in the literature is about $0.7 \%$ in pediatric cancer patients [1]. It is most commonly reported in cases of leukemia among hematological malignancies and solid tumors during their treatment with chemotherapeutic agents. The most common preceding factor is hypertension and presenting symptoms are seizures and altered mental status [2]. The pathophysiology behind this clinical syndrome is edema mainly of subcortical white matter resulting predominantly due to vasogenic and cytotoxic sequelae of hypertension and immunosuppressive therapies on cerebral blood flow, blood-brain barrier, and autoregulation [2,3]. The diagnosis is based on characteristic clinical and radiological findings [1-3].

The clinical context and the judgment of the clinician are crucial to make the correct diagnosis [3]. Thus, in resourcelimited settings or non-availability of the gold standard imaging to support the diagnosis, PRES can be a diagnosis of exclusion. To avoid complications and ensure complete neurological recovery, early recognition and intensive care are very essential in

\section{Access this article online}

Received - 05 May, 2021

Initial Review - 22 May, 2021

Accepted - 28 May, 2021

DOI: $10.32677 /$ IJCR.2021.v07.i06.005 children with PRES [4]. The case that we had encountered was a 2-year-old male child, under evaluation for a large retroperitoneal mass arising from the right adrenal gland that later revealed to be a pediatric adrenocortical tumor on a biopsy report.

\section{CASE REPORT}

A 2-year-old male child weighing $19 \mathrm{~km}$ with a history of gradually increasing abdominal distension for the past 4-5 months with no other constitutional symptoms was brought to the outpatient department (OPD) by his parents. The patient was the youngest of all three siblings, was born by normal vaginal delivery at full term, and had undergone essential immunization for his age. There was no history of malignancy in the family.

The physical, psychosocial, and developmental milestones were all in the normal range for the age of the child. The patient had stable vitals and normal general physical examination. The local examination revealed hirsutism, the presence of pubic hairs, and penile enlargement was denoted stage intravenous (IV) of Tanner's staging classification of secondary sexual characteristics. The abdominal examination revealed a firm mass palpable mass $10 \mathrm{~cm}$ below the costal margin.

Correspondence to: Dr. Vishal Bhatnagar, Department of Surgical Intensive Care Unit, Rajiv Gandhi Cancer Institute and Research Center, Rohini, New Delhi, India. E-mail: drvishalbhatnagar@yahoo.com

(C) 2021 Creative Commons Attribution-NonCommercial 4.0 International License (CC BY-NC-ND 4.0). 
The computed tomography scan (CT) whole abdomen and the triphasic liver study revealed a large heterogeneous mass of size $138 \mathrm{~mm} \times 117 \mathrm{~mm} \times 128 \mathrm{~mm}$ arising from the right adrenal gland compressing and displacing the right lobe of the liver superiorly with multiple abdominopelvic lymph nodes enlargement and nodular lesions were in the bilateral lung parenchyma (Fig. 1). The blood investigation reports were hemoglobin $11.7 \mathrm{~g} \%$, total leukocyte count $10,700 / \mathrm{mm}^{3}$, platelet count $460,000 / \mathrm{mm}^{3}$, prothrombin time $14.2 \mathrm{~s}$, INR 1.05 , serum urea $12.7 \mathrm{~g} \%$, creatinine $0.2 \mathrm{mg} \%$, serum bilirubin $0.23 \mathrm{mg} \%$, aspartate transaminase $252 \mathrm{IU} / \mathrm{L}$, alanine transaminase $336 \mathrm{IU} / \mathrm{L}$, alkaline phosphatase $213 \mathrm{IU} / \mathrm{L}$, serum albumin $3.6 \mathrm{~g} \%$, serum sodium, and potassium 138 and $4.7 \mathrm{mEq} / \mathrm{L}$, respectively.

The patient was admitted and planned for a CT-guided biopsy from abdominal mass under sedation. On the morning of the procedure, the pre-operative vitals of the patient were noted. The patient was afebrile with a heart rate of (HR) 136/min, respiratory rate (RR) of 20/min, blood pressure (BP) $90 / 60 \mathrm{mmHg}$, and oxygen saturation on pulse oximeter $\left(\mathrm{SPO}_{2}\right) 99 \%$ on room air. The procedure was successful and uneventful under a sedative dose of the combination of IV propofol, ketamine, and fentanyl. The emergence from the sedation was smooth and complete. The patient was shifted to the recovery area near the procedure room under continuous monitoring of $\mathrm{SPO}_{2}$, and intermittent monitoring of BP, and RR.

Suddenly, the patient started crying vigorously, on examination, HR was $128 / \mathrm{min}$ and BP was $189 / 103 \mathrm{mmHg}$ on the monitor. The patient was administered IV injection paracetamol $300 \mathrm{mg}$ and fentanyl $20 \mu \mathrm{g}$ considering procedure-related pain. The patient was shifted to the intensive care unit (ICU) for monitoring. By the time patient reached ICU, he was awake, lying comfortably in his mother's lap with a HR of 128/min, BP of 140/100 mmHg, RR of $25 / \mathrm{min}$, and $\mathrm{SPO}_{2}$ of $98 \%$ on room air. The patient was kept under close monitoring and observation. IV fluid at $50 \mathrm{ml} / \mathrm{h}$ of ringer's lactate was started keeping a long fasting period in view.

Till the time patient was being stabilized and comforted, he had sudden onset generalized tonic-clonic seizure for a few seconds followed by unresponsiveness and abnormal respiratory pattern. On examination, a surge in BP to $>190 / 105 \mathrm{mmHg}$ and tachycardia with HR $>150 /$ min was observed. A bolus of IV injection of labetalol $1 \mathrm{mg}$ was given immediately and repeated every 1-2 min till BP was $<140 / 95 \mathrm{mmHg}$. The patient was turned to a recovery position to protect the airway. Oxygenation and assisted ventilation through pediatric Ambu bag and mask

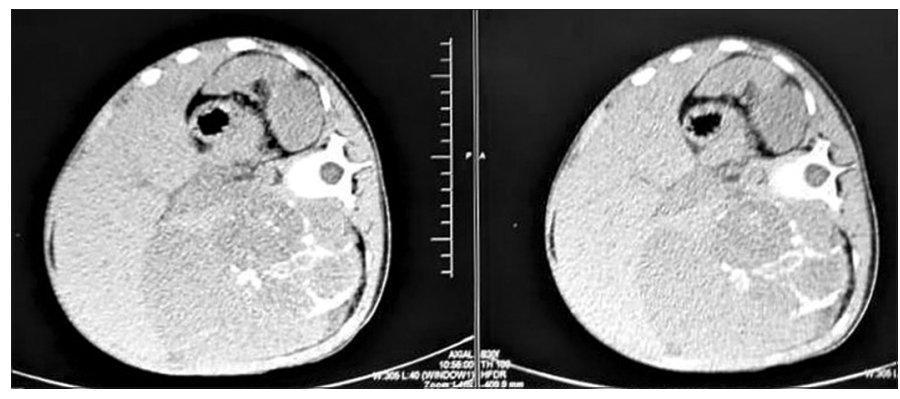

Figure 1: Computed tomography abdomen was initiated. In view of sustained and refractory hypertension, infusion of glyceryl trinitrate (GTN) was started at $5 \mu \mathrm{g} / \mathrm{kg} / \mathrm{min}$ and injection furosemide $20 \mathrm{mg}$ IV stat was given.

The patient had to be intubated because of altered sensorium, low Glasgow Coma Scale (GCS $<8$ ), and recurrent seizures to protect the airway from aspiration. The left radial arterial line was placed for continuous BP monitoring. The benzodiazepine (midazolam) $2 \mathrm{mg}$ was administered intravenously for immediate control of seizures followed by a loading dose of phenytoin $400 \mathrm{mg}$ IV in saline over $20 \mathrm{~min}$. The supportive ICU care was continued as per the standard protocol alongside antihypertensive measures. The patient kept sedated with low-dose fentanyl and propofol and BP targeted at $<140 / 80 \mathrm{mmHg}$ with a continuation of GTN and labetalol infusion. The target BP gradually lowered to $<110 / 70 \mathrm{mmHg}$ within several hours.

Injection NTG infusion was discontinued after $24 \mathrm{~h}$ and labetalol infusion tapered off gradually. A tablet of amlodipine $1.25 \mathrm{mg}$ twice daily and a tablet of prazosin $0.5 \mathrm{mg}$ thrice daily were also added to the antihypertensive regimen through Ryle's tube. The non-contrast CT scan brain showed normal study (Fig. 2) and electroencephalography was inconclusive. The transthoracic echocardiography also revealed a normal study. Gradually over $48 \mathrm{~h}, \mathrm{BP}$ was under control with antihypertensives and no seizure recurrence within the past $24 \mathrm{~h}$. The sedation was discontinued, the patient gradually weaned off from the mechanical ventilation and extubated.

The patient was fully awake there was no neurological deficit. The patient was discharged on the same day on request by parents after observation of $6 \mathrm{~h}$ on triple antihypertensives regimen (tab. labetalol $25 \mathrm{mg}$ QID, tab. amlodipine $1.25 \mathrm{mg} \mathrm{BD}$, and tab. prazosin $0.5 \mathrm{mg}$ TDS) and an antiepileptic drug (tab. phenytoin $50 \mathrm{mg}$ BD). The patient was advised to come for follow-up in pediatric oncology OPD. The patient did not appear for follow-up and could not be traced the due to prevailing COVID-19 pandemic situation.

\section{DISCUSSION}

The PRES is a rare occurrence in pediatric patients with solid tumors. There are few recent case reports of PRES in pediatric patients with the micronodular adrenocortical disease and Cushing's syndrome [5], pheochromocytoma [6], Wegener's granulomatosis [7], and chronic kidney disease (CKD) patient on hemodialysis [8]. The incidence of hypertensive PRES in children

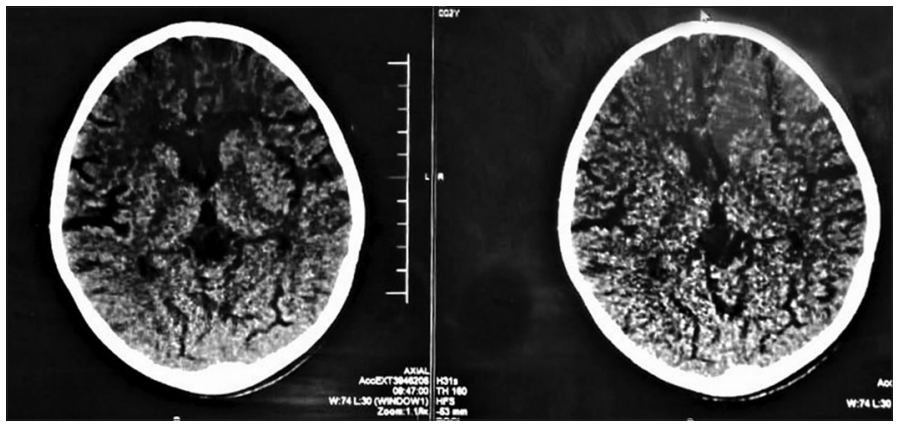

Figure 2: Non-contrast computed tomography brain 
is also a rare phenomenon [6], but the antecedent cause in our patient and all of these cases were pronounced hypertension and uremia along with hypertension in CKD patients.

The acute onset of neurological symptoms following a significant and sustained rise of BP well above the $99^{\text {th }}$ percentile for our patient by his age and height [9] helped in establishing the diagnosis in favor of PRES by the multidisciplinary team. The symptoms and signs such as seizures and altered sensorium that were the presenting feature in our patient and most of the other reported cases in the literature are not specific to PRES and can be encountered in many other neurological conditions. The differential diagnosis could be autoimmune or paraneoplastic encephalitis, central nervous system vasculitis, malignancy, or tumor (lymphoma, gliomatosis cerebri, and metastatic disease), infectious encephalopathy, etc. $[3,10]$.

The NCCT brain was unremarkable and magnetic resonance imaging (MRI) brain could not be performed for this patient due to the logistic and technical issues. MRI brain has more sensitivity and specificity in detecting abnormalities in the initial scan as compared to CT brain, $98 \%$ and $46 \%$, respectively [1]. The diagnosis of PRES in our patient was mainly based on the clinical context and judgment of the treating clinical team. Although the confirmation of PRES by brain MRI is the gold standard, the entity may also exist without abnormalities on brain imaging [10]. In the literature also, it is suggested that a patient with at least one acute neurological symptom (seizures, confusion, headache, or visual disturbances) and at least one risk factor (severe hypertension/BP fluctuations, chemotherapy, or autoimmune disorder) should be suspected to have PRES on clinical grounds irrespective of the brain imaging findings [3].

The principles of the management of PRES that we followed were also similar to the previous case reports that included intensive care requirements, antihypertensives, antiepileptics, and supportive care [6-9]. There are no specific protocols for prescribing antiepileptics or antihypertensive drugs in PRES management, but the mainstay of management is the treatment of hypertension and control of seizures with antiepileptic drugs [1-3,11]. The patient was planned for evaluation in subsequent visits but did not appear for follow-up and could not be traced back. Adrenocortical tumors are very rare in pediatric patients and are associated with a poor prognosis if malignant [12].

\section{CONCLUSION}

The PRES in pediatric patients is linked with a high mortality rate of up to $16 \%$ and has risks of potential neurological complications.
The clinical judgment, meticulous, and prompt management of this condition are a very essential step to save life and prevention of any potential neurological sequelae. The diagnosis of PRES is mainly based on characteristic clinical features and specific findings on MRI. In resource-limited institutions or facilities, the clinical context and judgment of the clinician are crucial for early diagnosis and management to prevent mortality and morbidity from this treatable clinical entity.

\section{REFERENCES}

1. Chen TH. Childhood posterior reversible encephalopathy syndrome: Clinicoradiological characteristics, managements, and outcome. Front Pediatr 2020;8:585.

2. de Laat P, Te Winkel ML, Devos AS, Catsman-Berrevoets CE, Pieters R, van den Heuvel-Eibrink MM. Posterior reversible encephalopathy syndrome in childhood cancer. Ann Oncol 2011;22:472-8.

3. Fugate JE, Rabinstein AA. Posterior reversible encephalopathy syndrome: Clinical and radiological manifestations, pathophysiology, and outstanding questions. Lancet Neurol 2015;14:914-25.

4. Chen TH, Lin WC, Tseng YH, Tseng CM, Chang TT, Lin TJ. Posterior reversible encephalopathy syndrome in children: Case series and systematic review. J Child Neurol 2013;28:1378-86.

5. Lodish M, Patronas NJ, Stratakis CA. Reversible posterior encephalopathy syndrome associated with micronodular adrenocortical disease and Cushing syndrome. Eur J Pediatr 2010;169:125-6.

6. Moorthy S, Subramaniam TK, Prabhu N, Sree KK, Nair R. Posterior reversible encephalopathy syndrome in a child with pheochromocytoma. Indian J Radiol Imaging 2002;12:321-4.

7. Sharma A, Tyagi A, Singal A. Posterior reversible encephalopathy syndrome in Wegener's granulomatosis: A rare occurrence. Indian J Anaesth 2020;64:538-9

8. Peter P, George A. Posterior reversible encephalopathy syndrome and the pediatric population. J Pediatr Neurosci 2012;7:136-8.

9. Bagga A, Jain R, Vijayakumar M, Kanitkar M, Ali U. Evaluation, and management of hypertension. Indian Pediatr 2007;44:103-21.

10. Faille LD, Fieuws S, van Paesschen W. Clinical predictors and differential diagnosis of posterior reversible encephalopathy syndrome. Acta Neurol Belg 2017;117:469-75.

11. Ndu IK, Ayuk AC, Onukwuli VO. Challenges of diagnosing pediatric posterior reversible encephalopathy syndrome in resource poor settings: A narrative review. Glob Pediatr Health 2020;7.

12. Sutter JA, Grimberg A. Adrenocortical tumors and hyperplasias in childhoodetiology, genetics, clinical presentation and therapy. Pediatr Endocrinol Rev 2006;4:32-9.

Funding: None; Conflicts of Interest: None Stated.

How to cite this article: Singh $\mathrm{R}$, Bhatnagar V. Management of posterior reversible encephalopathy syndrome in a pediatric patient with a rare adrenocortical tumor after computed tomography-guided biopsy. Indian J Child Reports. 2021;7(6):235-237. 